\title{
A micromechanics-based damage model for the strength prediction of composite laminates
}

\author{
PP Camanho*, JA Mayugo§, P Maimís, CG Dávila** \\ *DEMEGI, Faculdade de Engenharia, Universidade do Porto, Portugal \\ §AMADE, University of Girona, Spain \\ **NASA Langley Research Center, Hampton, VA, U.S.A.
}

\begin{abstract}
The prediction of the failure of composite laminates subjected to multi-axial loading in regions with mild stress gradients is of considerable interest for some aerospace applications such as pressurized vessels. In such components, which are devoid of notches or other stress concentrations, matrix cracks can accumulate prior to the localization of damage along a narrow fracture path.

The prediction of the onset of ply damage can be accomplished by using ply-level failure criteria [1]-[2]. To predict ultimate failure of a laminate the 'ply-discount' method is normally used. The ply discount method reduces by an empirical amount the elastic properties of a damaged ply as a function of the type of damage predicted. Such an approach is not rigorous for the simulation of the progressive accumulation of transverse matrix cracks.

The main objective of the current work is to develop an alternative method to predict the onset of ply damage and ultimate failure of a laminate. The failure criteria used to predict damage onset are the LaRC03 failure criteria [2].

A micromechanical model of a laminate containing matrix transverse cracks under multi-axial loading is proposed (Figure 1). The model proposed can predict the increased strength of a ply when it is embedded in a multidirectional laminate [3], and it relates the multi-axial strain state to the density of transverse matrix cracks.

Based on the micromechanical analyses, a new model is formulated in the framework of damage mechanics. The characteristics of the constitutive model, e.g. the free energy, the damage evolution functions and the loading-unloading
\end{abstract} conditions are based on the micromechanical model.

The algorithm for the integration of the constitutive equation and the implementation of the model in computational models based on the Finite Element Method or in the Classical Lamination Theory are presented. Several examples relating the applied loads to the stiffness of a multidirectional laminate are presented (Figure 2).

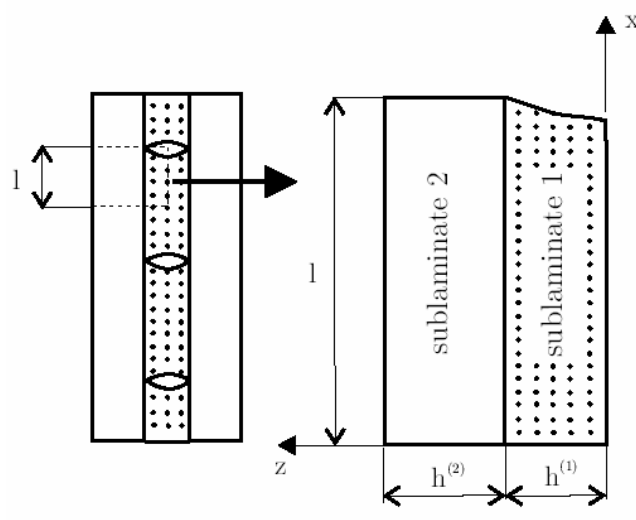

Fig. 1 Representative volume element of a laminate with matrix cracks

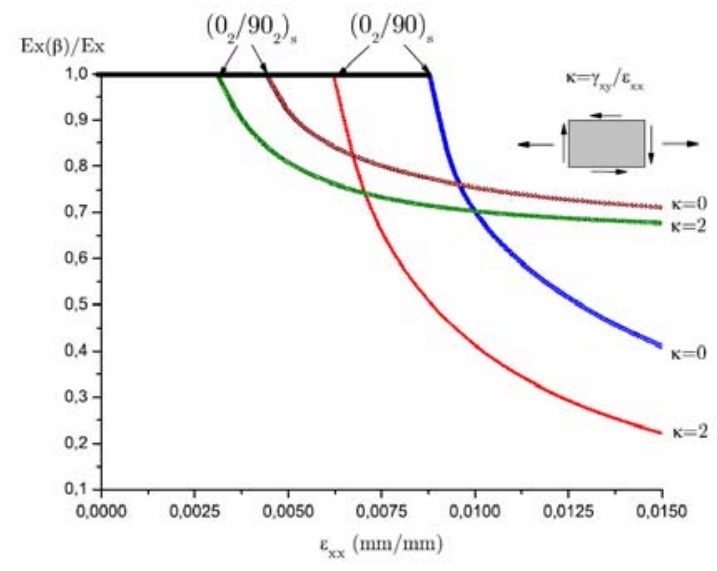

Fig. 2 Relation between applied strain and axial modulus of the composite.

[1] Soden, P.D.; Hinton, M.J., and Kaddour, A.S. A comparison of the predictive capabilities of current failure theories for composite laminates, Composites Science and Technology, Vol.58, pp. 1225-1254, 1998.

[2] Dávila, C.G., Camanho, P.P., and Rose, C.A. Failure criteria for FRP laminates, Journal of Composite Materials, Vol. 39, No. 4, pp. 323-345, 2005.

[3] Camanho, P.P. Dávila, C.G. et. al. Prediction of in-situ strengths and matrix cracking in composites under transverse tension and in-plane shear, Composites-Part A, in press, 2005. 


\title{
A MICROMECHANICS-BASED DAMAGE MODEL FOR THE STRENGTH PREDICTION OF COMPOSITE LAMINATES
}

\author{
Pedro P. Camanho ${ }^{1}$, Joan A. Mayugo ${ }^{2}$, Pere Maimí ${ }^{2}$ and Carlos G. Dávila ${ }^{3}$ \\ ${ }^{1}$ DEMEGI, Faculdade de Engenharia, Universidade do Porto \\ Rua Dr. Roberto Frias, 4200-465, Porto, Portugal \\ e-mail: pcamanho@fe.up.pt \\ 2 AMADE, Escola Politècnica Superior, Universitat de Girona \\ Campus Montilivi, 17071 Girona, Spain \\ e-mail: \{pere.maimi,ja.mayugo\}@udg.es \\ ${ }^{3}$ NASA Langley Research Center \\ Hampton, VA, U.S.A. \\ e-mail: Carlos.G.Davila@nasa.gov
}

Keywords: Continuum Damage Mechanics, Fracture, Composite Materials.

\begin{abstract}
A new damage model based on a micromechanical analysis of cracked $\left[ \pm \theta^{\circ} / 90_{n}^{\circ}\right]_{s}$ laminates subjected to multiaxial loads is proposed. The model predicts the onset and accumulation of transverse matrix cracks in uniformly stressed laminates, the effect of matrix cracks on the stiffness of the laminate, as well as the ultimate failure of the laminate. The model also accounts for the effect of the ply thickness on the ply strength. Predictions relating the elastic properties of several laminates and multiaxial loads are presented.
\end{abstract}




\section{INTRODUCTION}

The aerospace industry is committed to improve the performance of aircraft whilst reducing emissions and weight. Such a goal can be achieved by the use of advanced polymer-based composite materials, that have excellent properties for aerospace applications, such as low density, and fatigue and corrosion resistance.

The design procedure used for advanced composite structures relies on a 'building-block' approach [1], where a large number of experimental tests are performed throughout the product development process. The use of improved analytical or numerical models in the prediction of the mechanical behavior of composite structures can significantly reduce the cost of such structures. Such models should predict the onset of material degradation, the effect of the noncritical damage mechanisms on the stiffness of the laminate, and ultimate structural failure.

Strength-based failure criteria are commonly used to predict failure in composite materials [2]-[7]. Failure criteria predict the onset of the several damage mechanisms occurring in composites and, depending on the laminate, geometry and loading conditions, may also predict structural collapse.

In multidirectional composite laminates, damage accumulates during the loading process. Final failure occurs as a result of damage accumulation and stress re-distribution. The ultimate failure load is higher than the initial damage predicted by strength-based failure criteria. Furthermore, stress- or strain-based failure criteria cannot represent size effects that occur in quasi-brittle materials [8].

Simplified models, such as the ply discount method where some scalar components of the stiffness tensor are reduced to approximately zero when damage is predicted, are often used by the industry to predict ultimate laminate failure. However, these methods cannot represent with satisfactory accuracy the progressive reduction of the stiffness of a laminate as a result of the accumulation of matrix cracks.

Constitutive laws based on Continuum Damage Mechanics (CDM) have been proposed to predict the material response, from the onset of damage up to final collapse [9]-[14]. Although the existing CDM models can predict accurately the evolution of damage, they usually rely on fitting parameters that need to be measured at laminate level, such as the critical values of thermodynamic forces [14].

Three-dimensional models based on CDM use material properties measured at the ply level. Damage mechanisms such as transverse matrix cracks are represented by strain-softening constitutive models and bands of localized deformation. The implementation of strain-softening models in finite element models causes convergence difficulties during the iterative solution procedure. Furthermore, strain-softening models require regularization techniques to provide mesh-independent solutions [15, 16].

Alternative methods based on the combination of elastic analysis of cracked plies and finite Fracture Mechanics provide the basis for an accurate representation of the response of composite materials [17]-[28]. Micromechanical models have been developed to predict the initiation and evolution of transverse matrix cracks under either in-plane shear or transverse tension. Generalizations of these models are required for the more usual case of multiaxial loading.

In order to predict ultimate failure, a micromechanical model needs to be used in combination with a fiber failure criterion. Furthermore, a general methodology to predict laminate strength should be able to predict matrix cracking under high values of transverse compression. These damage mechanisms usually correspond to the final failure of laminates under uniform states of stress [6]. 
The objective of this work is to define a new damage model based on micromechanical models of transverse matrix cracks to predict the onset and evolution of transverse matrix cracks under multiaxial loading. A new constitutive model is derived based on the thermodynamics of irreversible processes. The model proposed herein represents transverse matrix cracks as distributed damage. The model is able to predict the onset and propagation of matrix transverse cracks under multiaxial loading as well as the final failure of uniformly stressed laminates where a periodic distribution of transverse matrix cracks can be assumed.

\section{MICROMECHANICAL MODEL}

The proposed continuum damage model is based on two major components: a set of stress based failure criteria and a micromechanical model of transverse matrix cracking in multidirectional laminates.

The failure criteria define the onset of transverse matrix cracking, i.e. the activation of the damage variables. A micromechanical model of transverse matrix cracks is required to define the evolution of the damage variables.

Several micromechanical models of transverse matrix cracks that have been proposed in the literature [19, 28] can be used within the framework developed here. The micromechanical model proposed by Tan and Nuismer [17, 18] accounts for the effects of the adjoining plies on the homogenized elastic properties of a cracked ply. This micromechanical model is the basis of the developments presented in this work.

Using the assumption of generalized plane strain, Tan and Nuismer [17, 18] developed a model able to relate the density of transverse matrix cracks in a central $90^{\circ}$ ply to the homogenized elastic properties of that ply. The model developed by Tan and Nuismer was used for the prediction of the evolution of transverse matrix cracks under either in-plane shear or transverse tensile stresses.

The laminates under investigation are symmetric and balanced with a $\left[ \pm \theta / 90_{\mathrm{n}}^{\circ}\right]_{s}$ layup containing a periodic distribution of transverse matrix cracks, as shown in Figure 1, The micromechanical analysis of a balanced symmetrical laminate requires the division of the laminate in two sub-laminates: the $90^{\circ}$ layers in the middle layer (sublaminate 1), and the outer plies (sublaminate 2).

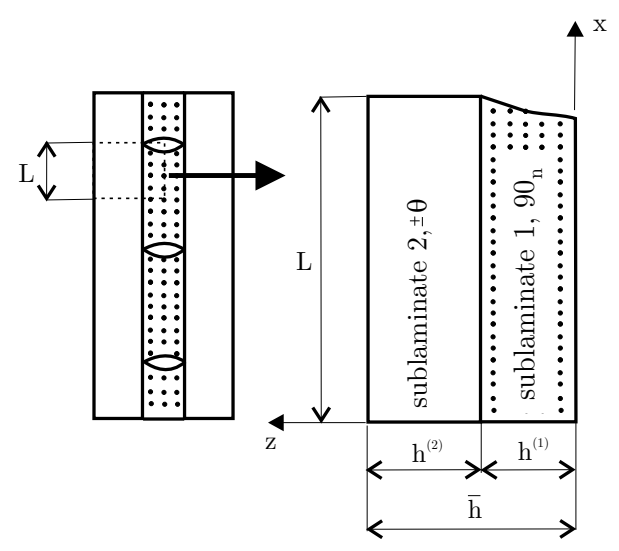

Figure 1: One-quarter of the laminate.

Sublaminate 1 is taken as transversely isotropic. The outer layers, sublaminate 2 , may contain several layers with different fiber orientations but must be orthotropic in the laminate global system ( $x, y$ and $z$ coordinates). 


\subsection{CONSTITUTIVE TENSOR}

The stiffness tensor of the balanced and symmetric laminate shown in Figure 1 can be written as a function of the density of transverse matrix cracks $(1 / L)$ in the sublaminate 1 :

$$
\overline{\mathbf{A}}(L)=\left[\begin{array}{ccc}
\bar{A}(L)_{11} & \bar{A}(L)_{12} & 0 \\
\bar{A}(L)_{21} & \bar{A}(L)_{22} & 0 \\
0 & 0 & \bar{A}(L)_{66}
\end{array}\right]
$$

where the terms $\bar{A}_{i j}(L), i, j=1,2,6$ are obtained from the Tan and Nuismer model [18].

The undamaged stiffness matrix $\overline{\mathbf{Q}}$ of the laminate is obtained from lamination theory using the undamaged stiffness matrix of sublaminate $1, \overline{\mathbf{Q}}^{(1)}$, and the stiffness matrix of sublaminate 2, $\overline{\mathbf{Q}}^{(2)}$ :

$$
Q_{i j}^{(1)}=\frac{1}{h^{(1)}}\left(\bar{h} \bar{Q}_{i j}-h^{(2)} Q_{i j}^{(2)}\right)
$$

with $\bar{h}=h^{(1)}+h^{(2)}$.

Assuming that the degradation due to the transverse matrix cracks only occur in sublaminate 1 , the damaged stiffness tensor of laminate 1 is given as:

$$
\bar{A}_{i j}^{(1)}(L)=\frac{1}{h^{(1)}}\left(\bar{h} \bar{A}_{i j}(L)-h^{(2)} Q_{i j}^{(2)}\right)
$$

where $\bar{A}_{i j}^{(1)}(L)(i, j=1,2,6)$ are the scalar components of the stiffness tensor of sublaminate 1 . These components are a function of the distance between transverse matrix cracks ( $L$, defined in Figure 1).

Having defined $\bar{A}_{i j}^{(1)}(L)$, it is possible to calculate the effective transverse modulus, Poisson ratio, and shear modulus of the $90^{\circ}$ ply:

$$
\begin{gathered}
\bar{E}_{2}^{(1)}(L)=\bar{E}_{x}^{(1)}(L)=\bar{A}_{11}^{(1)}(L)-\frac{\left[\bar{A}_{12}^{(1)}(L)\right]^{2}}{\bar{A}_{22}^{(1)}(L)} \\
\bar{v}_{21}^{(1)}(L)=\bar{v}_{x y}^{(1)}(L)=\frac{\bar{A}_{12}^{(1)}(L)}{\bar{A}_{22}^{(1)}(L)} \\
\bar{G}_{12}^{(1)}(L)=\bar{G}_{x y}^{(1)}(L)=\bar{A}_{66}^{(1)}(L)
\end{gathered}
$$

The quotient $\frac{v_{21}}{E_{2}}$ is not a function of damage. This observation is in agreement with several other models, such as the ones proposed by Laws et al. [23, 24], and Nguyen [25].

The plane stress compliance tensor of the damaged sublaminate $1, \mathbf{H}^{(1)}$, only contains two components that depend on the density of transverse matrix cracks: $H_{22}^{(1)}(L)$ and $H_{66}^{(1)}(L)$. The tensor $\mathbf{H}^{(1)}$, is established as a function of the density of transverse matrix cracks, $L$, as:

$$
\mathbf{H}^{(1)}=\left[\begin{array}{ccc}
\frac{1}{E_{1}} & -\frac{v_{21}}{E_{2}} & 0 \\
-\frac{v_{12}}{E_{1}} & H_{22}^{(1)}(L) & 0 \\
0 & 0 & H_{66}^{(1)}(L)
\end{array}\right]
$$


with:

$$
\begin{gathered}
H_{22}^{(1)}(L)=\frac{1}{\bar{E}_{2}^{(1)}(L)}=\frac{\bar{A}_{22}^{(1)}(L)}{\bar{A}_{11}^{(1)}(L) \bar{A}_{22}^{(1)}(L)-\left[\bar{A}_{12}^{(1)}(L)\right]^{2}} \\
H_{66}^{(1)}(L)=\frac{1}{\bar{G}_{12}^{(1)}(L)}=\frac{1}{\bar{A}_{66}^{(1)}(L)}
\end{gathered}
$$

The evolution of the homogenized elastic properties of the cracked ply (sublaminate 1) as a function of $\beta=1 /(2 L)$ is shown in Figure 2 for a $\left[ \pm 25^{\circ} / 90_{3}^{\circ}\right]_{s}$ laminate with the material properties shown in Table 1 .

\begin{tabular}{cccc}
\hline \hline $\mathrm{E}_{1}(\mathrm{GPa})$ & $\mathrm{E}_{2}(\mathrm{GPa})$ & $\mathrm{G}_{12}(\mathrm{GPa})$ & $v_{12}$ \\
\hline 163.4 & 11.9 & 6.2 & 0.30 \\
\hline \hline
\end{tabular}

Table 1: Elastic properties of typical carbon/epoxy composite.

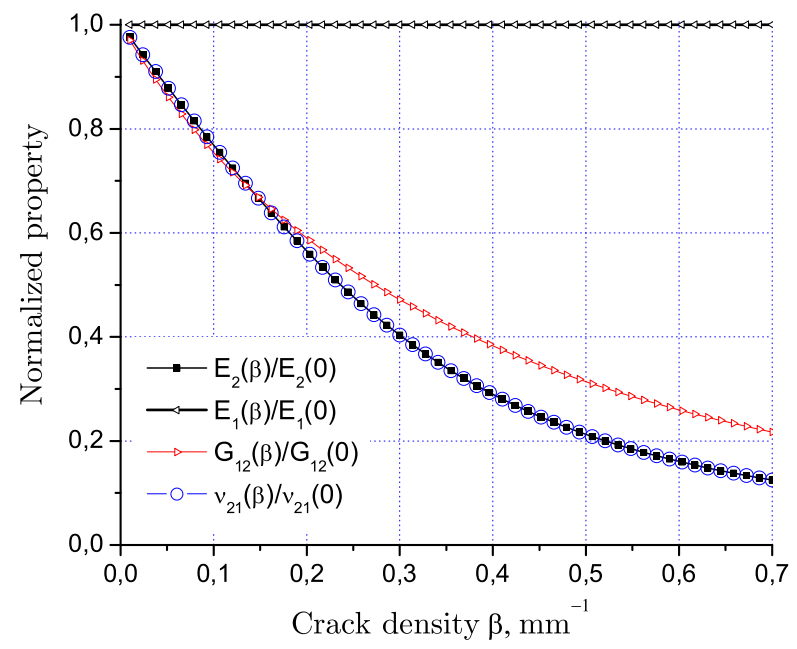

Figure 2: Effective elastic properties of the $90^{\circ}$ ply as a function of the density of matrix cracks.

Figure 2 shows that the Young modulus of sublaminate $1, E_{1}$, is not affected by the presence of transverse matrix cracks. Furthermore, the curves $E_{2}(\beta) / E_{2}$ and $v_{21}(\beta) / v_{21}$ are coincident (Figure 2).

\subsection{ONSET OF MATRIX TRANSVERSE CRACKS}

The onset of transverse matrix cracking in a ply under the combined effect of in-plane shear stresses and transverse tensile stresses needs to be predicted using an appropriate failure criterion. The failure criterion should be established in terms of the actual strengths of a ply when it is embedded in a multidirectional laminate (in-situ strengths). 


\subsubsection{In-situ strengths}

A methodology to predict the onset of matrix transverse cracking must be able to predict the in-situ strengths of a ply. Both the transverse tensile and in-plane shear strengths of a ply embedded in a multidirectional laminate are higher than the corresponding values measured in unidirectional laminates [29].

The thick models described in [30] are used. The tensile transverse in-situ strengths of sublaminate (1) is [30]:

$$
\begin{gathered}
\text { For a thin embedded ply: } Y_{\mathrm{T}}=\sqrt{\frac{8 G_{\mathrm{Ic}}}{\pi t \Lambda_{22}^{o}}} \\
\text { For a thick ply: } Y_{\mathrm{T}}=1.12 \sqrt{2} Y_{\mathrm{T}}^{\mathrm{ud}}
\end{gathered}
$$

where $Y_{\mathrm{T}}^{\mathrm{ud}}$ is the tensile transverse strength measured in a unidirectional test specimen, $t$ is the thickness of sublaminate $1, G_{\text {Ic }}$ is the mode I intralaminar fracture toughness and $\Lambda_{22}^{\circ}$ is defined as:

$$
\Lambda_{22}^{\circ}=2\left(\frac{1}{E_{2}}-\frac{v_{21}^{2}}{E_{1}}\right)
$$

The in-situ shear strengths are obtained as [29]:

$$
S_{\mathrm{L}}=\sqrt{\frac{\left(1+\chi \phi G_{12}^{2}\right)^{1 / 2}-1}{3 \chi G_{12}}}
$$

where $\chi$ is the shear response factor defined in [29], and the parameter $\phi$ is calculated according to the configuration of the ply:

$$
\begin{aligned}
\text { For a thick ply: } & \phi=\frac{12\left(S_{L}^{\mathrm{ud}}\right)^{2}}{G_{12}}+18 \chi\left(S_{L}^{\mathrm{ud}}\right)^{4} \\
\text { For a thin ply: } & \phi=\frac{48 G_{\mathrm{IIc}}}{\pi t}
\end{aligned}
$$

where $S_{L}^{\mathrm{ud}}$ is the shear strength measured using an unidirectional test specimen and $G_{\text {IIc }}$ is the mode II fracture toughness.

\subsubsection{Failure criterion for the prediction of transverse cracking under multiaxial loading}

In general, a ply represented by sublaminate 1 in Figure 1 is subjected to transverse tensile stresses and in-plane shear stresses. Under pure in-plane shear or pure transverse tension, the onset of transverse matrix cracking is predicted by comparing the components of the stress tensor with the respective in-situ strengths (defined in the previous section).

Under multiaxial loading, it is necessary to use a failure criterion to predict the onset of matrix cracking. The LaRC04 [4, 5] failure criteria are a function of the components of the stress tensor and in-situ strengths. For transverse tension, the criterion used is:

$$
(1-g) \frac{\sigma_{22}}{Y_{\mathrm{T}}}+g\left(\frac{\sigma_{22}}{Y_{\mathrm{T}}}\right)^{2}+\left(\frac{\sigma_{12}}{S_{\mathrm{L}}}\right)^{2}-1 \leq 0 \text { with } \sigma_{22} \geq 0
$$


where $g=\frac{G_{\mathrm{Ic}}}{G_{\mathrm{IIc}}} \cdot G_{\mathrm{IIc}}$ is the mode II component of the fracture toughness associated with matrix transverse cracking.

Under moderate values of transverse compression, the cracks propagate along the laminate thickness direction, and the LaRC04 failure criterion is:

$$
\frac{1}{S_{L}}\left\langle\left|\sigma_{12}\right|+\eta^{L} \sigma_{22}\right\rangle-1 \leq 0 \text { with } \sigma_{22}<0
$$

where $\eta^{L}$ is the coefficient of longitudinal influence defined in [4, 5].

\subsection{Evolution of matrix transverse cracks under multiaxial loading}

\subsubsection{Transverse tension}

Tan and Nuismer [18] obtained a closed-form expression that defines the evolution of transverse matrix cracks under uniaxial stress states (either transverse tension or in-plane shear).

To predict failure under multiaxial loading, i.e. when the lamina is simultaneously under tensile and in-plane shear strains, $\varepsilon_{x x}$ and $\gamma_{x y}$ respectively, it is necessary to derive a relation between the density of transverse matrix cracks and the applied multiaxial strain state. It is assumed that the relation between the tensile and shear strains is constant throughout the loading history:

$$
\kappa=\frac{\gamma_{x y}}{\varepsilon_{x x}} \text { with } \varepsilon_{x x}>0
$$

where $\kappa$ is the multiaxial strain ratio.

Consider a ply with crack density $L$, as shown in Figure 3 a).

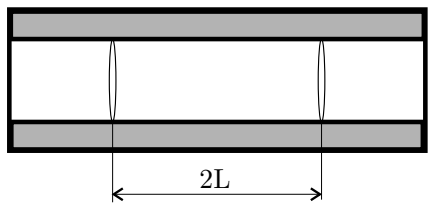

a) Crack density $2 \mathrm{~L}$

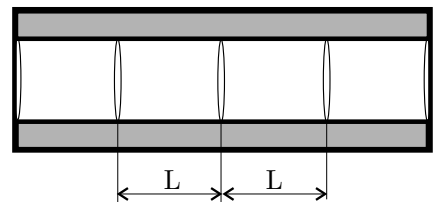

b) Crack density L

Figure 3: Progression of transverse matrix cracks.

The strain energy in a laminate cell of length $2 L$ subjected to transverse tension and in-plane shear just prior to fracture, $U_{2 L}$, can be established as a function of the strain tensor and of the crack density as:

$$
U_{2 L}=2 \bar{h} L b\left[E_{x}(L) \varepsilon_{x x}^{2}+A_{66}(L) \gamma_{x y}^{2}\right]
$$

where $E_{x}(L)$ is the axial stiffness of the laminate with a crack density of $1 /(2 L)$ and $b$ is the specimen width; $A_{66}(L)$ is the laminate effective shear stiffness corresponding to a crack density of $1 /(2 L), 2 \bar{h}$ and $2 L$ are the laminate thickness and the distance between two consecutive transverse matrix cracks, respectively.

After the propagation of transverse matrix cracks, Figure $3 \mathrm{~b}$ ), the strain energy in the original unit cell of length $2 L$ is:

$$
U_{L}=2 \bar{h} L b\left[E_{x}\left(\frac{L}{2}\right) \varepsilon_{x x}^{2}+A_{66}\left(\frac{L}{2}\right) \gamma_{x y}^{2}\right]
$$


where $E_{x}(L / 2)$ and $A_{66}(L / 2)$ are respectively the axial stiffness and the laminate effective shear stiffness corresponding to a crack density defined by $L$.

The energy required to generate a new matrix crack in a ply equals the loss of strain energy of the laminate [18]. Therefore, the difference between equation (18) and equation (19) is equal to the energy released by the sublaminate 1 :

$$
\begin{gathered}
\Delta U=U_{2 L}-U_{L}= \\
=2 \bar{h} L b\left\{\left[E_{x}(L)-E_{x}\left(\frac{L}{2}\right)\right] \varepsilon_{x x}^{2}+\left[A_{66}(L)-A_{66}\left(\frac{L}{2}\right)\right] \gamma_{x y}^{2}\right\} \\
\Delta U=2 h^{(1)} b G_{c}=2 h^{(1)} b G_{I}+2 h^{(1)} b G_{I I}
\end{gathered}
$$

where $G_{c}$ is the mixed-mode fracture toughness of sublaminate 1 under tensile (mode I) and shear (mode II) loading. From (20) and (21):

$$
\left[E_{x}(L)-E_{x}\left(\frac{L}{2}\right)\right] \varepsilon_{x x}^{2}+\left[A_{66}(L)-A_{66}\left(\frac{L}{2}\right)\right] \gamma_{x y}^{2}=\frac{h^{(1)} G_{c}}{\bar{h} L}
$$

Using the definition of $\kappa$ given in (17), equation (22) can be re-written as a function of the strains:

$$
\left\{\left(E_{x}(L)-E_{x}\left(\frac{L}{2}\right)\right)+\kappa^{2}\left[A_{66}(L)-A_{66}\left(\frac{L}{2}\right)\right]\right\} \varepsilon_{x x}^{2}=\frac{h^{(1)} G_{c}}{\bar{h} L}
$$

or:

$$
\left\{\frac{1}{\kappa^{2}}\left[E_{x}(L)-E_{x}\left(\frac{L}{2}\right)\right]+\left[A_{66}(L)-A_{66}\left(\frac{L}{2}\right)\right]\right\} \gamma_{x y}^{2}=\frac{h^{(1)} G_{c}}{\bar{h} L}
$$

The relations between the normal and shear strains and the crack density are obtained as:

$$
\begin{gathered}
\varepsilon_{x x}=\sqrt{\frac{h^{(1)} G_{c}}{\bar{h} L} \frac{1}{\left[E_{x}(L)-E_{x}\left(\frac{L}{2}\right)\right]+\kappa^{2}\left[A_{66}(L)-A_{66}\left(\frac{L}{2}\right)\right]}} \\
\gamma_{x y}=\sqrt{\frac{h^{(1)} G_{c}}{\bar{h} L} \frac{\kappa^{2}}{\left[E_{x}(L)-E_{x}\left(\frac{L}{2}\right)\right]+\kappa^{2}\left[A_{66}(L)-A_{66}\left(\frac{L}{2}\right)\right]}}=\kappa \varepsilon_{x x}
\end{gathered}
$$

Equations (25) and (26), are established as a function of the mixed-mode fracture toughness $G_{c}$ that needs to be defined.

The mixed-mode fracture toughness is defined in terms of the mode I and mode II components as:

$$
G_{c}=G_{I}+G_{I I}
$$

From (27) and (22):

$$
\begin{aligned}
\frac{h^{(1)} G_{I}}{\bar{h} L} & =\left[E_{x}(L)-E_{x}\left(\frac{L}{2}\right)\right] \varepsilon_{x x}^{2} \\
\frac{h^{(1)} G_{I I}}{\bar{h} L} & =\left[A_{66}(L)-A_{66}\left(\frac{L}{2}\right)\right] \gamma_{x y}^{2}
\end{aligned}
$$


Dividing equations (28) and (29) by (23) and (24), respectively:

$$
\begin{aligned}
A_{I} & =\frac{G_{I}}{G_{c}}=\frac{\left[E_{x}(L)-E_{x}\left(\frac{L}{2}\right)\right]}{\left[E_{x}(L)-E_{x}\left(\frac{L}{2}\right)\right]+\kappa^{2}\left[A_{66}(L)-A_{66}\left(\frac{L}{2}\right)\right]} \\
A_{I I} & =\frac{G_{I I}}{G_{c}}=\frac{\kappa^{2}\left[A_{66}(L)-A_{66}\left(\frac{L}{2}\right)\right]}{\left[E_{x}(L)-E_{x}\left(\frac{L}{2}\right)\right]+\kappa^{2}\left[A_{66}(L)-A_{66}\left(\frac{L}{2}\right)\right]}
\end{aligned}
$$

$A_{I}$ and $A_{I I}$ are the ratios between the mode I and mode II components of the energy release rate and the mixed-mode fracture toughness.

The criterion proposed by Hahn [31] for the prediction of transverse matrix cracking under transverse tensile and in-plane shear loads is used:

$$
(1-g) \sqrt{\frac{G_{\text {IIc }}}{G_{\text {Ic }}}}+g \frac{G_{\mathrm{I}}}{G_{\text {Ic }}}+\frac{G_{\text {II }}}{G_{\text {IIc }}}=1
$$

Substituting equations (30) and (31) into (32) gives:

$$
(1-g) \sqrt{\frac{A_{I} G_{c}}{G_{\mathrm{Ic}}}}+g \frac{A_{I} G_{c}}{G_{\mathrm{Ic}}}+\frac{\left(1-A_{I}\right) G_{c}}{G_{\mathrm{IIc}}}=1
$$

The positive real solution of $(33)$ is:

$$
G_{c}=G_{\mathrm{IIc}}+\frac{A_{I}}{2} \frac{\left(G_{\mathrm{Ic}}-G_{\mathrm{IIc}}\right)^{2}}{G_{\mathrm{Ic}}}\left(1-\sqrt{1+\frac{4}{A_{I}} \frac{G_{\mathrm{Ic}} G_{\mathrm{IIc}}}{\left(G_{\mathrm{Ic}}-G_{\mathrm{IIc}}\right)^{2}}}\right)
$$

where $A_{I}$ depends on the density of transverse matrix cracks, $\beta$, and on the multiaxial strain ratio, $\kappa$ (equation (30)).

Figure 4 shows the relation between the crack density $\beta$ and $\varepsilon_{x x}$ for different multiaxial strain ratios. A $\left[ \pm 25^{\circ} / 90_{3}^{\circ}\right]_{s}$ laminate with the elastic properties shown in Table 1 is used in the predictions.

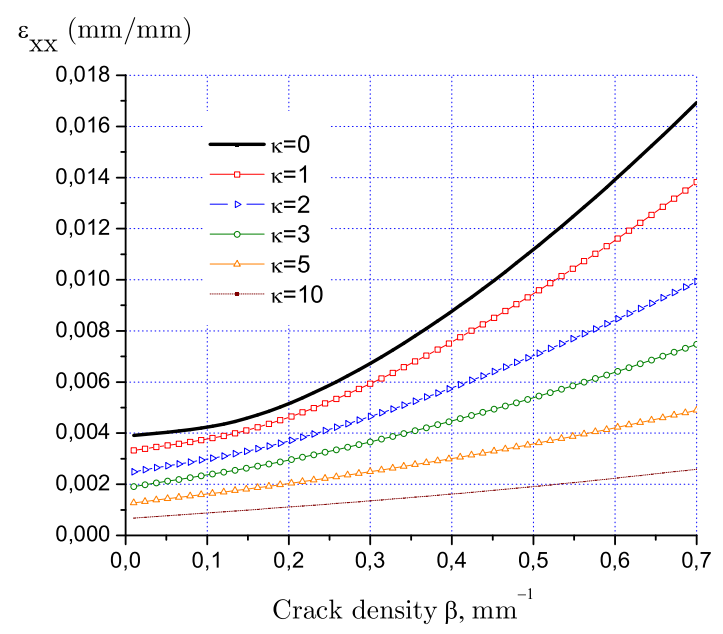

Figure 4: Relation between applied strain $\left(\varepsilon_{x x}\right.$ and $\left.\kappa\right)$ and density of matrix cracks. 
The effects of multiaxial strain states on the crack density are clearly shown in Figure 4. It can be observed in Figure 4 that the density of transverse matrix cracks increases with the multiaxial strain ratio for a fixed value of $\varepsilon_{x x}$.

\subsubsection{Transverse compression}

Transverse matrix cracks created by a combination of transverse compression and in-plane shear close under the effect of the compressive transverse stress. When a crack closes, its faces can transmit normal tractions but shear tractions may cause slippage between the crack faces. Therefore, it can be assumed that transverse matrix cracks only affect the shear stiffness of a laminate.

Following the procedure described in the previous section, the energy released by the sublaminate 1 is:

$$
\Delta U=2 \bar{h} L b \gamma_{x y}^{2}\left[A_{66}(L)-A_{66}\left(\frac{L}{2}\right)\right]=2 h^{(1)} b G_{\text {IIc }}
$$

The relation between the shear strain and the crack density is obtained as:

$$
\gamma_{x y}=\sqrt{\frac{h^{(1)} G_{\text {IIc }}}{\bar{h} L\left[A_{66}(L)-A_{66}\left(\frac{L}{2}\right)\right]}}
$$

\section{MICROMECHANICS-BASED DAMAGE MODEL}

\subsection{Constitutive model}

A damage model able to represent the onset and accumulation of a periodic distribution of transverse matrix cracks should yield a compliance tensor similar to the one obtained from the micromechanical model, equation (7). An appropriate damage model can be developed by defining the Gibbs free energy per unit volume, $\Psi_{G}$, as:

$$
\begin{aligned}
\Psi_{G}= & \frac{1}{2}\left[\frac{\sigma_{11}^{2}}{E_{1}}+\frac{\sigma_{22}^{2}}{\left(1-d_{2}\right) E_{2}}+\frac{\sigma_{12}^{2}}{\left(1-d_{6}\right) G_{12}}-\left(\frac{v_{21}}{E_{2}}+\frac{v_{12}}{E_{1}}\right) \sigma_{11} \sigma_{22}\right]+ \\
& +\left(\alpha_{11} \sigma_{11}+\alpha_{22} \sigma_{22}\right) \Delta T+\left(\beta_{11} \sigma_{11}+\beta_{22} \sigma_{22}\right) \Delta M
\end{aligned}
$$

where $E_{1}, E_{2}, v_{12}, v_{21}$ and $G_{12}$ are the in-plane elastic orthotropic properties of a unidirectional lamina. The subscript 1 denotes the longitudinal (fiber) direction and 2 denotes the transverse (matrix) direction. $d_{2}$ and $d_{6}$ are damage variables associated with transverse matrix cracking. $\alpha_{11}$ and $\alpha_{22}$ are the coefficients of thermal expansion in the longitudinal and transverse directions, respectively. $\beta_{11}$ and $\beta_{22}$ are the coefficients of hygroscopic expansion in the longitudinal and transverse directions, respectively. $\Delta T$ and $\Delta M$ are respectively the changes in temperature and moisture content from the stress-free state.

The proposed model distinguishes between active $\left(d_{2+}\right)$ and passive damage $\left(d_{2-}\right)$ variables, corresponding to the opening or closure of transverse matrix cracks under load reversal respectively. To account for the active damage under either opening or closure, the following equation is proposed:

$$
d_{2}=d_{2+} \frac{\left\langle\sigma_{22}\right\rangle}{\left|\sigma_{22}\right|}+d_{2-} \frac{\left\langle-\sigma_{22}\right\rangle}{\left|\sigma_{22}\right|}
$$


where the McCauley operator $\langle x\rangle$ is defined as $\langle x\rangle:=\frac{1}{2}(x+|x|)$.

The damage variables $d_{2}$ and $d_{6}$ can be related to the density of transverse matrix cracks using the micromechanical model previously described.

The constitutive model is obtained as:

$$
\varepsilon=\frac{\partial \Psi_{G}}{\partial \sigma}=\mathbf{H}: \sigma+\Delta T \alpha+\Delta M \beta
$$

where the compliance tensor $\mathbf{H}$ is:

$$
\mathbf{H}=\frac{\partial^{2} \Psi_{G}}{\partial \sigma^{2}}=\left[\begin{array}{ccc}
\frac{1}{E_{1}} & -\frac{v_{21}}{E_{2}} & 0 \\
-\frac{v_{12}}{E_{1}} & \frac{1}{\left(1-d_{2}\right) E_{2}} & 0 \\
0 & 0 & \frac{1}{\left(1-d_{6}\right) G_{12}}
\end{array}\right]
$$

The compliance tensor is established in terms of the damage variables and is similar to the compliance tensor derived in the micromechanical model.

Using (7) and (40) the damage variables $d_{2}$ and $d_{6}$ can be expressed in terms of the crack density $\beta$ as:

$$
\begin{aligned}
& d_{2+}=1-\frac{1}{E_{2} H_{22}(\beta)} \\
& d_{6}=1-\frac{1}{G_{12} H_{66}(\beta)}
\end{aligned}
$$

The functions $H_{22}(\beta)$ and $H_{66}(\beta)$ are obtained from equations (8) and (9) with $\beta=1 /(2 L)$.

The condition of positive energy dissipation is established as a function of the thermodynamic forces, $\mathbf{Y}$, and as a function of the time derivative of the damage variables, $\dot{\mathrm{d}}$, and it is given by:

$$
\begin{array}{r}
\Xi=\mathbf{Y} \cdot \dot{\mathbf{d}}=\frac{\partial \Psi_{G}}{\partial \mathbf{d}} \cdot \dot{\mathbf{d}} \geq 0 \\
\Xi=\frac{\sigma_{22}^{2}}{2\left(1-d_{2+}\right)^{2} E_{2}} \dot{d}_{2+}+\frac{\sigma_{12}^{2}}{2\left(1-d_{6}\right)^{2} G_{12}} \dot{d}_{6} \geq 0
\end{array}
$$

where $\Xi$ is the rate of energy dissipation per unit volume.

The condition of positive dissipation, when interpreted from a micromechanical point of view, establishes that the density of transverse matrix cracks can only increase or remain constant.

\subsubsection{Damage activation functions}

Transverse matrix cracks are predicted using two scalar functions, $F_{k}(k=2+, 2-)$, established in terms of the effective stress tensor $\tilde{\sigma}^{t}$, and of the damage threshold value, $r^{t}$ :

$$
F_{k}:=\phi_{k}\left(\tilde{\sigma}^{t}\right)-r^{t} \leq 0
$$

where $t$ is the current time. The initial threshold value, $r^{\circ}$, is equal to 1 , and the following condition must be satisfied in order to fulfill the Second Principle of Thermodynamics:

$$
\dot{r} \geq 0
$$


Damage onset occurs when any of the functions $\phi_{k}\left(\tilde{\sigma}^{t}\right)$ reaches the initial damage threshold value of 1 . The functions $\phi_{k}\left(\tilde{\sigma}^{t}\right)$ used to predict transverse matrix cracking are based on the LaRC04 failure criteria previously proposed by the authors [4, 5]. The damage activation functions are used to predict the onset of transverse matrix cracks lying in ply thickness direction, as shown in Figure 1.

Transverse matrix cracks lying in the direction of the ply thickness occur under transverse tension $\left(\sigma_{22} \geq 0\right)$, or under moderate values of transverse compression and in-plane shear $\left(\sigma_{22}<0\right)$.

For high values of transverse compression, the matrix fractures lie along a plane that is inclined at an angle $\alpha$ to the direction of the ply thickness. Inclined fracture planes caused by transverse compression induce delamination between the plies. This damage mechanism is usually catastrophic in uniformly stressed composites where local redistribution to more lightly loaded regions of the structure cannot occur [6]. Therefore, laminate failure can be assumed to occur when matrix cracking under high values of transverse compression is predicted.

The damage activation function used to predict matrix cracking under transverse tension $\left(\tilde{\sigma}_{22}^{t} \geq 0\right)$ and in-plane shear is defined as:

$$
F_{2+}:=\phi_{2+}\left(\tilde{\sigma}^{t}\right)-r^{t} \leq 0
$$

with:

$$
\phi_{2+}\left(\tilde{\sigma}^{t}\right)=\sqrt{(1-g) \frac{\tilde{\sigma}_{22}^{t}}{Y_{\mathrm{T}}}+g\left(\frac{\tilde{\sigma}_{22}^{t}}{Y_{\mathrm{T}}}\right)^{2}+\left(\frac{\tilde{\sigma}_{12}^{t}}{S_{\mathrm{L}}}\right)^{2}}
$$

The damage activation function used to predict matrix cracking with $\alpha=0^{\circ}$ under moderate values of transverse compression $\left(\tilde{\sigma}_{22}^{t}<0\right)$ and in-plane shear is defined as:

$$
F_{2-}:=\phi_{2-}\left(\tilde{\sigma}^{t}\right)-r^{t} \leq 0
$$

with:

$$
\phi_{2-}\left(\tilde{\sigma}^{t}\right) \underset{\alpha=0^{\circ}}{=} \frac{1}{S_{L}}\left\langle\left|\tilde{\sigma}_{12}^{t}\right|+\eta^{L} \tilde{\sigma}_{22}^{t}\right\rangle
$$

The equations proposed can predict accurately transverse matrix transverse cracking. The failure envelope, or initial elastic limit, predicted using equations (46) and (48), and the corresponding experimental data are shown in Figure 5. 


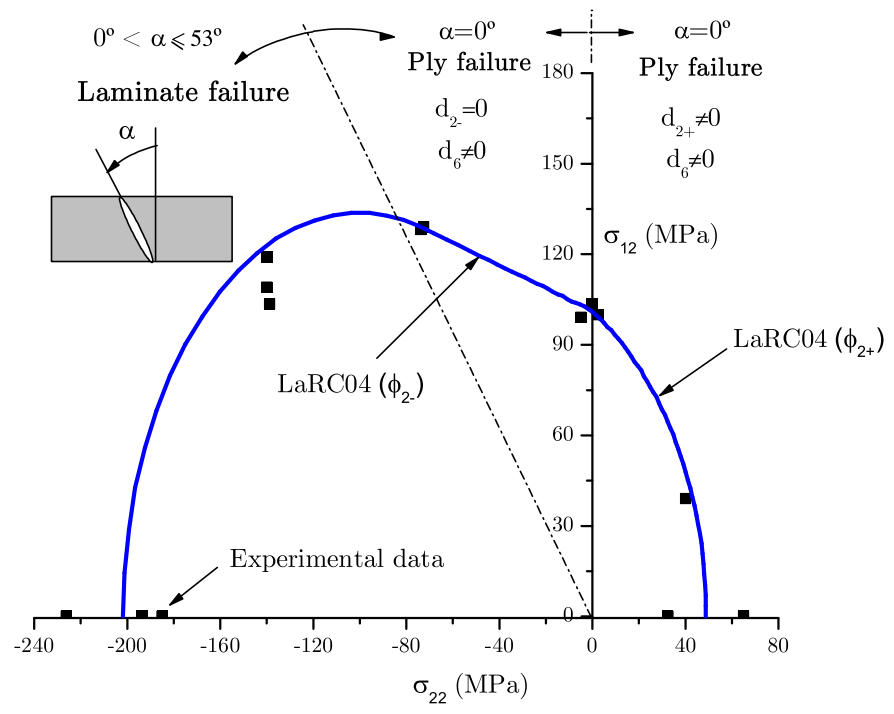

Figure 5: Comparison between predictions and experimental data [32] and definition of ply or laminate failure domains.

Figure 5 also shows the domain of validity of the model: the damage model is only defined for fracture angles $\alpha=0^{\circ}$. For $\alpha>0^{\circ}$, the laminate is assumed to fail.

\subsubsection{Damage evolution functions}

Using the principle of strain equivalence, the effective stress tensor can be written as a function of the strain and undamaged stiffness tensors:

$$
\tilde{\sigma}=\mathbf{C}^{o}: \varepsilon
$$

The strain tensor is established in terms of the density of transverse cracks and the multiaxial strain ratio $\kappa$, equations $(25 \cdot 26)$. Therefore, all terms in the damage threshold functions can be formulated as a function of the density of transverse matrix cracks and of the multiaxial strain ratio:

$$
F_{k}=f\left(\beta^{t}, \kappa^{t}\right)
$$

For a given state of strain at time $t$, the multiaxial strain ratio is a dependent variable that can be easily defined using equation (17) in material coordinates:

$$
\kappa^{t}=\frac{\gamma_{12}^{t}}{\varepsilon_{11}^{t}}
$$

The density of transverse matrix cracks is a state variable. Therefore, it is necessary to define an evolution law subjected to thermodynamic restrictions.

The first condition to be satisfied is the requirement of positive dissipation:

$$
\dot{\beta} \geq 0
$$


Furthermore, it is necessary to define the conditions for the evolution of the elastic domain:

$$
\begin{gathered}
F_{k}\left(\tilde{\sigma}^{t}, \beta^{t}\right) \leq 0 \\
\dot{\beta} F_{k}\left(\tilde{\sigma}^{t}, \beta^{t}\right)=0
\end{gathered}
$$

Equations (53)-(55) are the Kuhn-Tucker conditions which ensure a consistent evolution of damage during loading and load reversals.

The evolution laws of the state variables are defined as:

$$
\begin{aligned}
\dot{\tilde{\sigma}} & =\frac{\partial \tilde{\sigma}}{\partial \varepsilon}: \dot{\varepsilon}=\frac{\partial \tilde{\sigma}}{\partial \varepsilon}:\left(\frac{\partial \varepsilon}{\partial \beta} \dot{\beta}+\frac{\partial \varepsilon}{\partial \kappa} \dot{\kappa}\right)=\mathbf{C}^{o}:\left(\frac{\partial \varepsilon}{\partial \beta} \dot{\beta}+\frac{\partial \varepsilon}{\partial \kappa} \dot{\kappa}\right) \\
\dot{r} & =\frac{\partial r}{\partial \beta} \dot{\beta}+\frac{\partial r}{\partial \kappa} \dot{\kappa}
\end{aligned}
$$

For a given loading state, the damage consistency condition must be applied to define the evolution of the internal variables. The consistency condition is defined as:

$$
F_{k}=0 \Rightarrow \dot{F}_{k}=\frac{\partial F_{k}}{\partial \tilde{\sigma}}: \dot{\sigma}+\frac{\partial F_{k}}{\partial r} \dot{r}=0
$$

From (46) and (48):

$$
\frac{\partial F_{k}}{\partial r}=-1
$$

Using (59) in (58):

$$
\dot{F}_{k}=\frac{\partial F_{k}}{\partial \tilde{\sigma}}: \dot{\sigma}-\dot{r}=\frac{\partial F_{k}}{\partial \tilde{\sigma}}: \mathbf{C}^{\mathrm{o}}: \dot{\varepsilon}-\dot{r}=0
$$

Using equations (56) and (57) in (60):

$$
\dot{F}_{k}=\frac{\partial F_{k}}{\partial \tilde{\sigma}}: \mathbf{C}^{\mathrm{o}}:\left(\frac{\partial \varepsilon}{\partial \beta} \dot{\beta}+\frac{\partial \varepsilon}{\partial \kappa} \dot{\kappa}\right)-\left(\frac{\partial r}{\partial \beta} \dot{\beta}+\frac{\partial r}{\partial \kappa} \dot{\kappa}\right)=0
$$

Taking into account that the micromechanical model proposed assumes a constant loading ratio, $\dot{\kappa}=0$ :

$$
\dot{F}_{k}=\frac{\partial F_{k}}{\partial \tilde{\sigma}}:\left(\mathbf{C}^{\mathrm{o}}: \frac{\partial \varepsilon}{\partial \beta}-\frac{\partial r}{\partial \beta}\right) \dot{\beta}=0
$$

The density of of transverse matrix cracks, $\beta$, is calculated from the integration of equation (62) using a numerical method, such as the return-mapping algorithm.

\section{LAMINATE FAILURE}

In the presence of stress concentrations the onset of fiber localized failure does not cause immediate structural collapse. Experimental results [33, 34] show that structural collapse is caused by the the progression of fiber fracture. Therefore, it is necessary to use a damage model that accounts for the stress re-distributions caused by fiber fractures.

The model proposed here assumes that for uniformly stressed laminates the onset of fiber fracture and delamination caused by high compressive transverse stresses triggers structural collapse. Therefore, laminate final failure is predicted when fiber failure or matrix cracking with $\alpha \neq 0^{\circ}$ occurs. 


\subsection{Fiber failure}

The criterion for fiber fracture under longitudinal tension $\left(\sigma_{11} \geq 0\right)$ is defined as [4, 5]:

$$
\mathrm{FI}_{1+}:=\frac{\sigma_{11}}{X_{\mathrm{T}}}-1 \leq 0
$$

where $X_{\mathrm{T}}$ is the ply tensile strength in the longitudinal direction.

The LaRC03 failure criterion for fiber kinking under longitudinal compression $\left(\sigma_{11}<0\right)$ is a function of the components of the stress tensor in a frame representing the fiber misalignment, $\sigma_{i j}^{(m)}[4,5]:$

$$
\begin{aligned}
& \sigma_{11}^{(m)}=\sigma_{11} \cos ^{2} \varphi+\sigma_{22} \sin ^{2} \varphi+2\left|\sigma_{12}\right| \sin \varphi \cos \varphi \\
& \sigma_{22}^{(m)}=\sigma_{11} \sin ^{2} \varphi+\sigma_{22} \cos ^{2} \varphi-2\left|\sigma_{12}\right| \sin \varphi \cos \varphi \\
& \sigma_{12}^{(m)}=-\sigma_{11} \sin \varphi \cos \varphi+\sigma_{22} \sin \varphi \cos \varphi+\left|\sigma_{12}\right|\left(\cos ^{2} \varphi-\sin ^{2} \varphi\right)
\end{aligned}
$$

where the fiber misalignment angle $\varphi$ is defined as [4]:

$$
\begin{aligned}
\varphi & =\frac{\left|\sigma_{12}\right|+\left(G_{12}-X_{\mathrm{C}}\right) \varphi^{c}}{G_{12}+\sigma_{11}-\sigma_{22}} \\
\varphi^{c} & =\tan ^{-1}\left[\frac{1-\sqrt{1-4\left(\frac{S_{\mathrm{L}}}{X_{\mathrm{C}}}+\eta^{L}\right)\left(\frac{S_{\mathrm{L}}}{X_{\mathrm{C}}}\right)}}{2\left(\frac{S_{\mathrm{L}}}{X_{\mathrm{C}}}+\eta^{L}\right)}\right]
\end{aligned}
$$

where $X_{\mathrm{C}}$ is the ply compressive strength in the longitudinal direction.

Depending on the sign of the in-plane transverse stress $\sigma_{22}^{(m)}$, the criteria for fiber kinking $\left(\sigma_{11}<0\right)$ are:

$$
\begin{aligned}
\mathrm{FI}_{1-} & :=\left\langle\frac{\left|\sigma_{12}^{(m)}\right|+\eta^{L} \sigma_{22}^{(m)}}{S_{\mathrm{L}}}\right\rangle-1 \leq 0, \sigma_{22}^{(m)}<0 \\
\text { or } \mathrm{FI}_{1-}: & =(1-g) \frac{\sigma_{12}^{(m)}}{Y_{\mathrm{T}}}+g\left(\frac{\sigma_{12}^{(m)}}{Y_{\mathrm{T}}}\right)^{2}+\left(\frac{\sigma_{12}^{(m)}}{S_{\mathrm{L}}}\right)^{2}-1 \leq 0, \sigma_{22}^{(m)} \geq 0
\end{aligned}
$$

\subsection{Matrix failure with $\alpha \neq 0^{\circ}$}

The failure criteria for matrix cracking under transverse compression $\left(\sigma_{22}<0\right)$ and in-plane shear and $\alpha \neq 0^{\circ}$ are defined as [4, 5]:

$$
\begin{array}{r}
\mathrm{FI}_{2-}:=\left(\frac{\tau_{e}^{T}}{\mathbf{S}_{\mathrm{T}}}\right)^{2}+\left(\frac{\tau_{e}^{L}}{\mathrm{~S}_{\mathrm{L}}}\right)^{2}-1 \leq 0,\left|\sigma_{11}\right| \leq \mathrm{Y}_{\mathrm{C}} \\
\mathrm{FI}_{2-}:=\left(\frac{\tau_{e}^{(m) T}}{\mathrm{~S}_{\mathrm{T}}}\right)^{2}+\left(\frac{\tau_{e}^{(m) L}}{\mathrm{~S}_{\mathrm{L}}}\right)^{2}-1 \leq 0,\left|\sigma_{11}\right|>\mathrm{Y}_{\mathrm{C}}
\end{array}
$$

where the effective shear stresses in the fracture plane are defined as: 


$$
\begin{aligned}
\tau_{e}^{T} & =\left\langle\left|\tau^{T}\right|+\eta^{T} \sigma_{n} \cos \theta\right\rangle \\
\tau_{e}^{L} & =\left\langle\left|\tau^{L}\right|+\eta^{L} \sigma_{n} \sin \theta\right\rangle
\end{aligned}
$$

where $\theta=\tan ^{-1}\left(\frac{-\left|\sigma_{12}\right|}{\sigma_{22} \sin \alpha}\right)$. The components of the stress tensor on the fracture plane are given by [4, 5]:

$$
\left\{\begin{array}{l}
\sigma_{n}=\sigma_{22} \cos ^{2} \alpha \\
\tau^{T}=-\sigma_{22} \sin \alpha \cos \alpha \\
\tau^{L}=\sigma_{12} \cos \alpha
\end{array}\right.
$$

The terms $\tau_{e}^{m T}$ and $\tau_{e}^{m L}$ are calculated from equations (71)-(73) using the relevant components of the stress tensor established in a frame representing the fiber misalignment, equation (64). The angle $\alpha$ is determined by maximizing the failure index $\mathrm{FI}_{2-}(\underline{69-70})$ using a simple iterative procedure.

The coefficients of transverse and longitudinal influence, $\eta^{T}$ and $\eta^{L}$, respectively, are [4, 5]:

$$
\begin{aligned}
\eta^{T} & =\frac{-1}{\tan 2 \alpha_{0}} \\
\eta^{L} & =-\frac{\mathrm{S}_{\mathrm{L}} \cos 2 \alpha_{0}}{\mathrm{Y}_{\mathrm{C}} \cos ^{2} \alpha_{0}}
\end{aligned}
$$

with $\alpha_{0} \approx 53^{\circ} . Y_{\mathrm{C}}$ is the ply compressive strength in the transverse direction.

\section{EXAMPLES}

The present damage model can be used in combination with classical lamination theory using stand-alone codes. Alternatively, the damage model can be implemented as a constitutive subroutine for the finite element method.

The damage model was implemented using a commercial symbolic computing software. The model was verified by calculating the response of several glass-epoxy laminates under uniaxial and multiaxial loads: $\left[ \pm 45^{\circ} / 90_{4}^{\circ}\right]_{s},\left[0_{2}^{\circ} / 90_{4}^{\circ}\right]_{s},\left[0_{2}^{\circ} / 90_{2}^{\circ}\right]_{s}$ and $\left[0_{2}^{\circ} / 90^{\circ}\right]_{s}$.

In all calculations performed, the ply thickness was taken as $0.144 \mathrm{~mm}$, and the temperature difference from the stress free condition was $-100^{\circ} \mathrm{C}$. The coefficients of thermal expansion in the longitudinal and transverse directions are $\alpha_{11}=7.43 \times 10^{-6} /{ }^{\circ} \mathrm{C}$ and $\alpha_{22}=22.4 \times 10^{-6} /{ }^{\circ} \mathrm{C}$, respectively. The remaining material properties used are shown in Tables 2 and 3 .

\begin{tabular}{ccccccc}
\hline \hline $\mathrm{E}_{1}(\mathrm{GPa})$ & $\mathrm{E}_{2}(\mathrm{GPa})$ & $\mathrm{G}_{12}(\mathrm{GPa})$ & $\mathrm{G}_{23}(\mathrm{GPa})$ & $v_{12}$ & $v_{23}$ & $\chi\left(10^{-8} \mathrm{MPa}^{-3}\right)$ \\
\hline 44.7 & 12.8 & 5.8 & 4.5 & 0.30 & 0.42 & 2.0 \\
\hline \hline
\end{tabular}

Table 2: Elastic properties of glass-epoxy [20] 


\begin{tabular}{cccc}
\hline \hline $\mathrm{Y}_{\mathrm{T}}^{\text {ud }}(\mathrm{MPa})$ & $\mathrm{S}_{\mathrm{L}}^{\text {ud }}(\mathrm{MPa})$ & $\mathrm{G}_{\mathrm{Ic}}\left(\mathrm{N} / \mathrm{mm}^{-1}\right)$ & $\mathrm{G}_{\text {IIc }}\left(\mathrm{N} / \mathrm{mm}^{-1}\right)$ \\
\hline 40.0 & 73.0 & 0.20 & 0.40 \\
\hline \hline
\end{tabular}

Table 3: Strengths and fracture toughnesses of glass-epoxy [20].

The predicted response of $\left[ \pm 40^{\circ} / 90_{4}^{\circ}\right]_{s}$ and $\left[0_{2}^{\circ} / 90_{4}^{\circ}\right]_{s}$, is shown in Figure 6 for two values of the multiaxial strain ratio: $\kappa=0$ and $\kappa=10$.

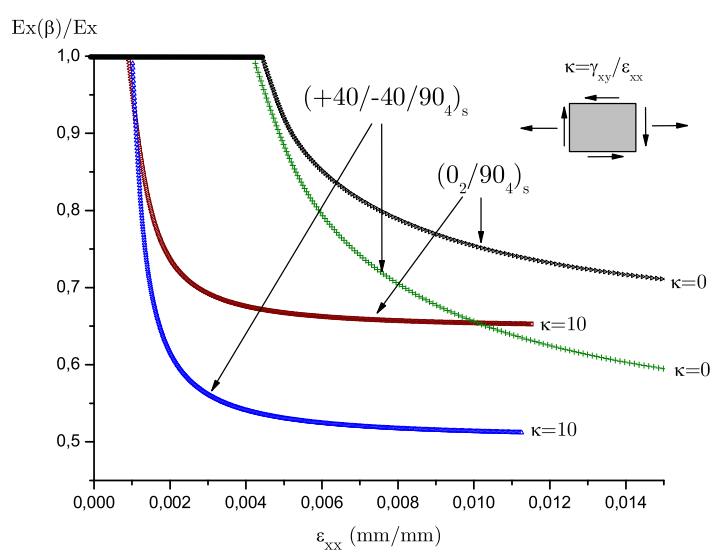

Figure 6: Relation between laminate modulus and applied strain for $\left[ \pm 45^{\circ} / 90_{4}^{\circ}\right]_{s}$ and $\left[0_{2}^{\circ} / 90_{4}^{\circ}\right]_{s}$ laminates.

Figure 6 shows that the rate of degradation of the elastic properties of the laminate is higher when the axial stiffness of the outer sublaminate decreases. The effect of multiaxial loading is also clear in Figure 6: as expected, the application of shear strains leads to a reduction of the extension corresponding to the onset of transverse matrix cracks and to a higher rate of degradation of the elastic properties of the laminate.

Figure 7 compares the response of $\left[0_{2}^{\circ} / 90_{4}^{\circ}\right]_{s},\left[0_{2}^{\circ} / 90_{2}^{\circ}\right]_{s}$ and $\left[0_{2}^{\circ} / 90^{\circ}\right]_{s}$ laminates for $\kappa=0$ and $\kappa=2$.

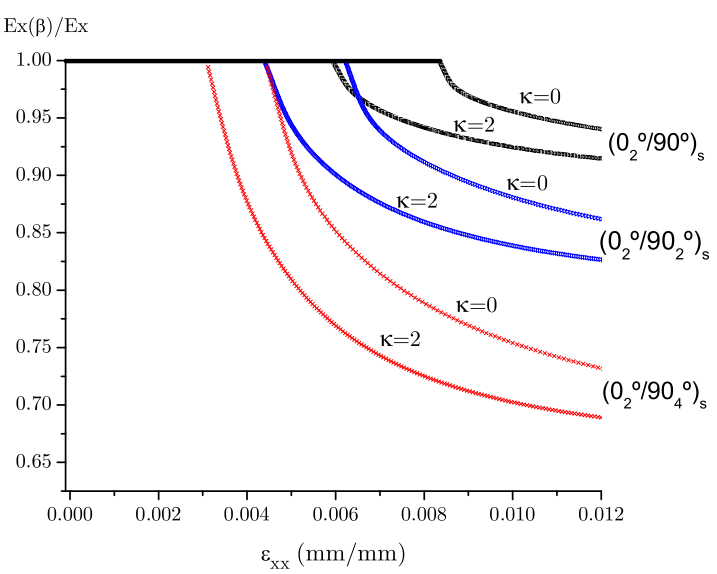

Figure 7: Relation between laminate modulus and applied strain for $\left[0_{2}^{\circ} / 90_{4}^{\circ}\right]_{s},\left[0_{2}^{\circ} / 90_{2}^{\circ}\right]_{s}$ and $\left[0_{2}^{\circ} / 90^{\circ}\right]_{s}$ laminates. 
The in-situ effect is shown in Figure 7: for $\kappa=0$, the strain corresponding to the onset of matrix cracking of the $\left[0_{2}^{\circ} / 90^{\circ}\right]_{s}$ laminate is 1.9 and 1.3 times higher than the strains of the $\left[0_{2}^{\circ} / 90_{4}^{\circ}\right]_{s}$ and $\left[0_{2}^{\circ} / 90_{2}^{\circ}\right]_{s}$ laminates respectively. Furthermore, the strain at the onset of matrix cracking of the $\left[0_{2}^{\circ} / 90^{\circ}\right]_{s}$ is 2.7 times higher than the ultimate transverse strain measured in an unidirectional test specimen.

\section{CONCLUSIONS}

A new, micromechanics-based, continuum damage model able to simulate the onset and propagation of transverse matrix cracks and final laminate failure is proposed. The model is applicable to $\left[ \pm \theta^{\circ} / 90_{n}^{\circ}\right]_{s}$ laminates, under multiaxial loading and uniform stresses or small stress gradients.

The model uses ply properties and does not require any tests performed at the laminate level to identify damage onset and evolution functions. The onset of damage is predicted using failure criteria and damage evolution laws are established from the micromechanical analysis of cracked plies.

The onset and accumulation of transverse matrix cracks are represented as a distributed damage mechanism. The onset of localization, which is triggered by either fiber fracture or matrix cracking with $\alpha \neq 0$, is assumed to cause a structural collapse.

The predictions show that the rate of degradation of the elastic properties increases when the stiffness of the outer sublaminate decreases. Decreasing the thickness of the $90^{\circ}$ plies also increases the degradation rate.

\section{Acknowledgements}

The financial support of the Portuguese Foundation for Science and Technology (FCT) under the project PDCTE/EME/50354/2003 is acknowledged by the second author. The present work has been partially funded by the Spanish Government under research project MAT2003-09768C03-001.

\section{REFERENCES}

[1] MIL-HDBK-17-3F, Military Handbook, Polymer Matrix Composites. U.S. Department of Defense, 2002.

[2] M.J. Hinton, A.S. Kaddour, and P. D. Soden, A comparison of the predictive capabilities of current failure theories for composite laminates, judged against experimental evidence. Composites Science and Technology, 62, 1725-1797, 2002.

[3] P.D. Soden, A.S. Kaddour, and M.J. Hinton, Recommendations for designers and researchers resulting from the world-wide failure exercise. Composites Science and Technology, 64, 589-604, 2004.

[4] C.G. Dávila and P.P. Camanho, Failure criteria for FRP laminates in plane stress, NASA/TM-2003-212663, National Aeronautics and Space Administration, 2003.

[5] S.T. Pinho, C.G. Dávila, P.P. Camanho, L. Iannucci and P. Robinson, Failure models and criteria for FRP under in-plane shear or three-dimensional stress states including shear non-linearity. NASA/TM-2005-213530, National Aeronautics and Space Administration, 2005 . 
[6] A. Puck and H. Schürmann, Failure analysis of FRP laminates by means of physically based phenomenological models, Composites Science and Technology, 58, 1045-1067, 1998.

[7] K.S. Liu, and S.W. Tsai, A progressive quadratic failure criterion for a laminate, Composites Science and Technology, 58, 1023-1032, 1998.

[8] Z.P. Bažant, Size effect. International Journal of Solids and Structures, 37, 69-80, 2000.

[9] P. Ladevèze, On a damage mechanics approach. Mechanics and Mechanisms of damage in composites and multi-materials. D. Batiste ed., 119-141, London, 1991.

[10] K.V. Williams, R. Vaziri and A. Poursartip. A physically based continuum damage mechanics model for thin laminated composite structures, International Journal of Solids and Structures, 40, 2267-2300, 2003.

[11] J. Varna, R. Joffe, N.V. Akshantala and R. Talreja, Damage in composite laminates with off-axis plies, Composites Science and Technology, 59, 2139-2147, 1999.

[12] D.H. Allen, C.E. Harris and S.E. Groves, A thermomechanical constitutive theory for elastic composites with distributed damage- I. theoretical development, International Journal of Solids and Structures, 23, 1301-1318, 1987.

[13] D.H. Allen, C.E. Harris and S.E. Groves, A thermomechanical constitutive theory for elastic composites with distributed damage- II. application to matrix cracking in laminated composites, International Journal of Solids and Structures, 23, 1319-1338, 1987.

[14] C.T. Herakovich, Mechanics of Fibrous Composites. Wiley, 1997.

[15] Z.P. Bažant and B.H. Oh, Crack band theory for fracture of concrete. Matériaux et Constructions, 16, 155-177, 1983.

[16] M. Jirásek, Modeling of localized damage and fracture in quasibrittle materials. Lecture Notes in Physics 568,17-29,P.A. Vermeer et al. ed., 2001.

[17] R.J. Nuismer and S.C. Tan, Constitutive relations of a cracked composite lamina, Journal of Composite Materials, 22, 306-321, 1988.

[18] S.C. Tan and R.J. Nuismer, A theory for progressive matrix cracking in composite laminates, Journal of Composite Materials, 23, 1029-1047, 1989.

[19] L.A. Berglund, J. Varna and J. Yuan, Effect of intralaminar toughness on the transverse cracking strain in cross-ply laminates. Advanced Composite Materials, 1, 225-234, 1991.

[20] R. Joffe, A. Krasnikovs and J. Varna, COD-based simulation of transverse cracking and stiffness reduction in (S/90n)s laminates, Composites Science and Technology, 61, 637656, 2001.

[21] R. Joffe and J. Varna, Analytical modeling of stiffness reduction in symmetric and balanced laminates due to cracks in 90 layers. Composites Science and Technology, 59, 16411652, 1999. 
[22] J. Varna, R. Joffe and R. Talreja, A synergistic damage-mechanics analysis of transverse cracking in $\left( \pm \theta / 90_{4}\right) \mathrm{s}$ laminates. Composites Science and Technology, 61, 657-665, 2001.

[23] G.J. Dvorak, N. Laws and M. Hejazi, Analysis of progressive matrix cracking in composite laminates I. thermoelastic properties of a ply with cracks, Journal of Composite Materials, 19, 216-234, 1985.

[24] N. Laws, G.J. Dvorak and M. Hejazi, Stiffness changes in unidirectional composites caused by crack systems, Mechanics of Materials, 2, 123-137, 1983.

[25] B.N. Nguyen, A three-dimensional modeling of transverse matrix cracking in laminated composites, Key Engineering Materials, 127, 1117-1126, 1997.

[26] G.A. Schoeppner and N.J. Pagano, 3-D thermoelastic moduli and saturation crack density for cross-ply laminates with transverse cracks, International Journal of Damage Mechanics, 8, 1-37, 1999.

[27] Z. Hashin, Analysis of cracked laminates: a variational approach, Mechanics of Materials, 4, 121-136, 1985.

[28] L.N. McCartney, G.A. Schoeppner and W. Becker, Comparison of models for transverse ply cracks in composite laminates, Composites Science and Technology, 60, 2347-2359, 20009.

[29] P.P. Camanho, C.G. Dávila, S.T. Pinho, L. Iannucci and P. Robinson, Prediction of insitu strengths and transverse matrix cracking in composites under transverse tension and in-plane shear, Composites-Part A, 37, 165-176, 2006.

[30] G.J. Dvorak and N. Laws, Analysis of first ply failure in composite laminates, Engineering Fracture Mechanics, 25, 763-770, 1986.

[31] H.T. Hahn, A mixed-mode fracture criterion for composite materials, Composites Technology Review, 5, 26-29, 1983.

[32] S.R. Swanson, A micro-mechanical model for in-situ compression strength of fiber composite laminates, Transactions of the American Society of Mechanical Engineers, Series H, Journal of Engineering Materials and Technology, 114, 8-12, 1992.

[33] P.P. Camanho, S. Bowron and F.L. Matthews, Failure mechanisms in bolted CFRP, Journal of Reinforced Plastics and Composites, 17, 205-233, 1998.

[34] P.P. Camanho, Application of numerical methods to the strength prediction of mechanically fastened joints in composite laminates. PhD Thesis, Centre for Composite Materials, Department of Aeronautics, Imperial College London, U.K., 1999. 